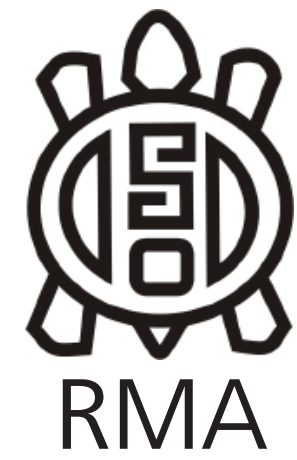

Dossier

\title{
Historias locales de plantas y personas. Nota sobre arqueo-etnobotánica del sitio Soria 2, sur de Yocavil
}

\author{
Local stories of plants and people. Note on archaeo-ethnobotany \\ site Soria 2 sur Yocavil
}

\author{
Natalia S. Petrucci* y Romina C. Spano** \\ *Laboratorio de Etnobotánica y Botánica Aplicada, Facultad de Ciencias Naturales y \\ Museo, UNLP. E- mail: nataliapetrucci@gmail.com

\begin{abstract}
** Centro Regional de Investigaciones Científicas y Transferencia Tecnológica de La Rioja (CRILAR) - CONICET. Instituto de las Culturas (IDECU), Museo Etnográfico "Juan B. Ambrosetti"- UBA-CONICET. E- mail: romina.spano@gmail.com
\end{abstract}

\begin{abstract}
Resumen
Este trabajo presenta una síntesis de las evidencias obtenidas a lo largo de 15 años de investigaciones en el sitio arqueológico Soria 2 (Valle de Yocavil, provincia de Catamarca), relativas a las identificaciones de vegetales usados en el pasado. Si bien el sitio fue objeto de numerosas aproximaciones plasmadas en tesis y artículos, hasta el momento la información concerniente a la utilización de especies vegetales no había sido amalgamada en una única contribución centrada en las interacciones entre personas y plantas. Teniendo como marco a la Arqueo-etnobotánica nos planteamos en esta oportunidad dos objetivos principales, bajo una mirada integradora. El primero es presentar el listado de especies asociadas a la ocupación temprana del sitio, a las cuales se identificó desde distintas aproximaciones: microrrestos y macrorrestos vegetales, y análisis químicos. El segundo objetivo es reconocer las prácticas que involucraron el uso de plantas, teniendo en cuenta la identificación taxonómica y poniendo en juego información erigida mediante otras líneas de evidencia. De este modo, es posible delinear las acciones y aspectos que conformaron la cotidianidad del grupo social que habitó en la vivienda obtención, preparación y consumo de alimentos, hilado, confección de utensilios, ritual, entre otras. Estas prácticas se considerarán bajo una perspectiva multiescalar. El propósito último, en definitiva, es construir un relato o historia local sobre las interacciones entre plantas y seres humanos, y a la vez aportar al estado de la cuestión sobre las comunidades tempranas -de inicios del primer milenio $A D-$, a través de un acercamiento a prácticas que intersectan aspectos como conocimiento, uso y manejo del entorno.
\end{abstract}

Palabras clave: Micro y macrorrestos vegetales; Interacciones entre personas y plantas; Historia local, Yocavil.

\begin{abstract}
This paper presents a synthesis of the evidences obteined from over 15 years of research at the archaeological site Soria 2 (Valle de Yocavil province of Catamarca) concerning the identification of the plants used in the past. Although the site has been was the subject of numerous approaches embodied in several thesis and articles, the information concerning the use of plant species has not been gathered in a single contribution focused on the interactions between people and plants. Within the framework of Archaeo-ethnobotany, we consider two main objectives, under an integrative multiescalar perspective. The first of them is to present a list of the species associated to the early occupation of the site, which were identified based on different approaches: plant microremains and macroremains, phytoliths studies and chemical analysis. The second objective is to recognize the practices involving the use of plants, taking into account their taxonomic identification and putting into play the information obtained through other lines of evidence. Thus, it is possible to delineate the actions and issues that shaped the daily life of the social group that inhabited the house: obtaining, preparing and consuming food, spinning, tool making, ritual practices, among others. These practices were considered under a multiscale perspective. The general aim, ultimately, is to build a local story about the interactions between plants and humans, contributing to the state of question about the early communities -beginning of the first millennium-, through an approach to those practices that intersect aspects such as knowledge, use and management of the environment.
\end{abstract}

Keywords: Plant microremains and macroremains; Interactions between people and plants; Local history; Yocavil.

Recibido 24-12-2019. Recibido con correcciones 11-06-2020. Aceptado 30-06-2020 


\section{Introducción}

Desde los inicios de las investigaciones en el sitio arqueológico Soria 2 (primer milenio de la Era, sur del valle de Santa María o Yocavil, Catamarca), la atención estuvo focalizada en dar a conocer los contextos de hallazgo, la cerámica asociada y la caracterización arquitectónica. Esto respondió a la falta de información contextual que persistía promediando la década del 2000 en el área bajo estudio. Si bien el sitio fue objeto de numerosas aproximaciones plasmadas en tesis y artículos, hasta el momento la información concerniente a la utilización de especies vegetales, obtenida a partir de distintas técnicas de recuperación, no había sido amalgamada en una única contribución con eje en las interacciones entre personas y plantas. Más bien, nuestras producciones académicas se centraron en la presentación de resultados puntuales.

Es sabido que el devenir de la producción de conocimiento se encuentra interceptado por factores diversos que inciden en la profundización de determinadas líneas de evidencia. Estos factores pueden ser desde las historias de los equipos de investigación, los intereses individuales de los miembros del grupo, la abundancia relativa de los vestigios arqueológicos recuperados según su materialidad, la disponibilidad de equipamiento, la vinculación con otros equipos de trabajo, etc.

En este trabajo presentamos una síntesis de la evidencia obtenida en el sitio Soria 2 relativa a la identificación de vegetales usados en el pasado a lo largo de 15 años de investigaciones. Desde una perspectiva arqueoetnobotánica, la cual aborda las relaciones entre los seres humanos y las plantas en el pasado, pretendemos interpretar a los restos arqueobotánicos como la materialidad que permite aproximarnos a la diversidad biocultural en el pasado. El punto de partida es entender a la diversidad biocultural como la diversidad biológica que resulta de prácticas de manejo humano, estando prácticas y plantas tan estrechamente unidas que la desaparición podría eventualmente causar la extinción de las otras (Maffi 2001). Esta diversidad es parte de modos de hacer situados localmente, los cuales se fundan en el conocimiento ambiental tradicional (Nazarea 1999). Este último es el resultado de una construcción comunitaria basada en la oralidad y se manifiesta en tecnologías y prácticas que se fundamentan y renuevan en la relación directa con el entorno; dando lugar al desarrollo de capacidades técnicas que serán gestionadas comunitaria o individualmente ante las contingencias que plantee el entorno biosocial y las distintas líneas de fuerza que atraviesen al mismo a lo largo del tiempo.

De esta manera planteamos dos objetivos principales: el primero es presentar el listado de plantas asociadas a la ocupación temprana -de inicios del primer milenio AD-, del sitio, a las cuales se identificaron desde distintas aproximaciones: observación de microrrestos vegetales, macrorrestos vegetales y análisis químicos. Se incluyen en este trabajo identificaciones que alcanzan diferentes niveles de la clasificación taxonómica (familia, género y especie).

El segundo objetivo es reconocer las prácticas que involucraron el uso de plantas, teniendo en cuenta la identificación taxonómica y considerando información erigida mediante otras líneas de evidencia, como el estudio de la alfarería, el lítico y la arquitectura. Nos servirá como herramienta para la integración de la información, la propuesta de Giménez (2001) quien desde la geografía humana amalgama distintas escalas espaciales de territorios para plantear una perspectiva multiescalar.

De manera simplificada podemos visualizar el modo subyacente de entender las interacciones entre los seres humanos y las plantas de la siguiente forma: los actores sociales representados por las plantas y personas, los rangos de acción espacial, y por último los eventos evidenciados a partir de la interpretación de los hallazgos.

El propósito, en definitiva, es construir un relato o historia local sobre las interacciones entre plantas y seres humanos, y a la vez aportar al estado de la cuestión sobre las comunidades tempranas, a través de un acercamiento a prácticas que intersectan aspectos como conocimiento, uso y manejo del entorno.

\section{Soria 2: emplazamiento y marco ambiental}

El sitio Soria 2, localizado entre las sierras del Aconquija y el sector sudoriental del valle de Yocavil (Figura 1.1), se asienta en una antigua mesada del tercer nivel pedemontano denominada Mesada de Andalhuala Banda (MAB) de la formación Caspinchango, sobre cuyo sector sur se emplaza el poblado actual de Andalhuala La Banda (Spano et al. 2014).

Las excavaciones permitieron definir dos recintos adosados subcuadrangulares Recinto 1 (R1) y Recinto 2 (R2), conformados por muros de líneas simples o dobles (Figura 1.2). En estos recintos se determinó un piso de ocupación con varios rasgos (fogón, agujeros de poste y un pozo de basura) y abundantes materiales culturales. En ambos recintos se hallaron entierros de infantes, depositados luego del abandono de la vivienda. Un fechado radiocarbónico de carbón asociado a un fogón

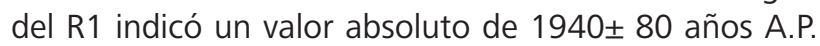
(L.P. 1541) (Palamarczuk et al. 2007).

Las precipitaciones anuales en el valle no superan los $200 \mathrm{~mm}$, con lluvias concentradas entre octubre y abril. La marcada amplitud de las precipitaciones y los valores de evapotranspiración que superan los $700 \mathrm{~mm}$ anuales genera un déficit hídrico que hace imposible realizar cualquier tipo de cultivos sin la asistencia de riego. El carácter oscilante de la temperatura es otro de los factores que configuran el clima semi-desértico del 


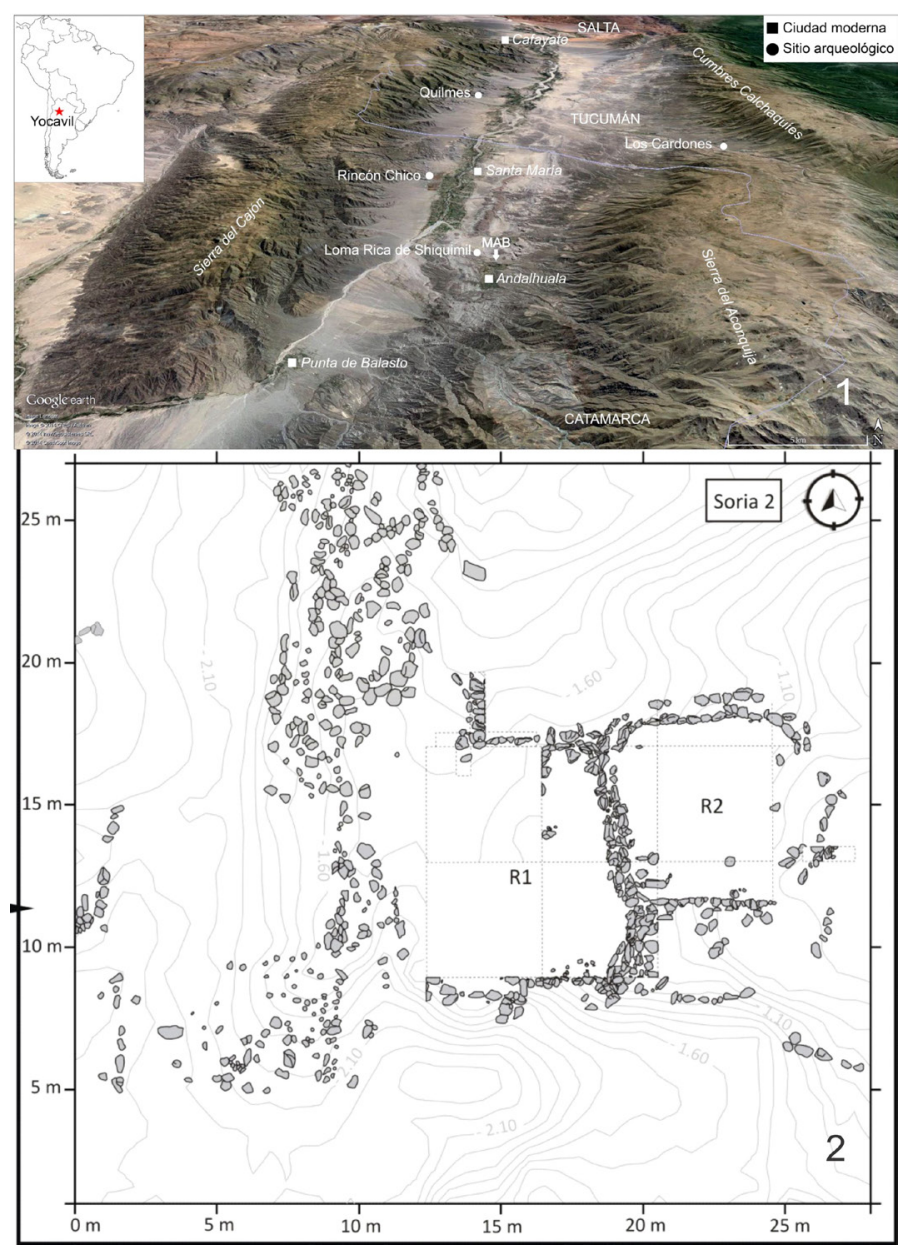

valle. El ambiente presenta insolación extrema con altas temperaturas en verano y bajas en invierno (Tineo 2005). La amplitud térmica diaria también es muy marcada, observándose muy altas temperaturas durante el día y muy bajas en la noche.

Fitogeográficamente el valle de Yocavil corresponde a la Región Neotropical, Dominio Chaqueño, incluyendo dos provincias: Prepuneña y del Monte. En la provincia del Monte se incluyen la estepa de Larrea divaricata, $L$. cuneifolia y L. nítida (larrea o jarillas) y arbustal abierto con predominio de especies caducifolias. La Prepuna se encuentra presente hacia el este del MAB, caracterizada por cactáceas columnares (Trichocereus sp.) de gran porte, siguiendo la clasificación de Cabrera (1971) se puede definir como una suerte de ecotono entre ambas provincias que integran el área.

\section{Integración de las líneas de investigación}

En este apartado exponemos los resultados obtenidos, a través de distintas técnicas, en relación a la identificación de restos botánicos. Para detalles acerca de los procedimientos seguidos en cada caso, por falta de espacio remitimos a las correspondientes publicaciones. Los materiales fueron recuperados bajo tres modalidades: (1) aislados, (2) en la matriz sedimentaria y (3) en el interior de artefactos.
Figura 1. Ubicación del sitio Soria 2 y otros sitios en el valle de Santa María. 2. Planta del sitio Soria 2 (tomado de Álvarez Larrain, 2014).

Figure 1. Location of the Soria 2 site and other sites in the Santa María Valley. 2. Plant 2 site Soria (taken from Alvarez Larrain, 2014).

Los análisis químicos se llevaron a cabo a partir de la aplicación de cromatografía gaseosa acoplada con espectrometría de masa al contenido carbonizado de un fragmento de hornillo de una pipa (Pipa 1), de pasta fina, color grisáceo y decoración geométrica en pintura roja post cocción, hallada en el pozo de basura del R1. Se detectó la presencia de dos alcaloides -Dimetiltriptamina $\mathrm{y}$-5-Hidroxidimetiltriptamina o bufotenina-, compuestos activos de Anadenanthera colubrina var. cebil (Rosso y Spano 2005). Los análisis fueron realizados por la Dra. Marta Maier, del Laboratorio de la Unidad de Microanálisis y Métodos Físicos Aplicados a la Química Orgánica (Facultad de Ciencias Exactas y Naturales de la Universidad de Buenos Aires).

Además, los contenidos remanentes de la Pipa 1 fueron objeto de análisis microscópico para la identificación histológica anatómica y taxonómica de microrrestos; se incluyó para este estudio de contenidos un segundo ejemplar de hornillo (Pipa 2) con decoración al pastillaje ornitomorfa, pintura post cocción y similares características tecnológicas. En la Pipa 1 se detectó fibras de aff. Gossypium sp. -una de las fibras teñida de rojo-, granos de almidón correspondientes a Zea mays y un tricoma ramificado de aff. Nicotiana sp. (Figura 2). En la Pipa 2 se identificó también fibras de aff. Gossypium sp., una de ellas con posibles marcas tecnológicas. Los estudios se llevaron a cabo en el Laboratorio de Etnobotánica y Botánica Aplicada, Facultad de Ciencias Naturales y Museo, UNLP (Andreoni et al. 2012).

También se realizaron estudios exploratorios de fitolitos sobre sedimentos procedentes del fogón. Los análisis se hicieron en el Laboratorio de Paleobotánica, Centro de Investigación Científica y Transferencia Tecnológica a la Producción, CONICET (Zucol 2005). Los restos fitolíticos están representados principalmente por fitolitos aislados, entre los cuales abundaron los tipos Estrobilolitas, Doliolitas, Prismatolita, Aculeolitas, y Securisaxum. Los fitolitos articulados resultaron escasos, correspondiéndose con conjuntos de células buliformes y/o células largas silicificadas. También se hallaron Globulolitas, Halteriolitas y Flabelolitas (Zucol 2005 Ms.). Algunos fitolitos poseían restos carbonosos en su matriz o en su superficie, de manera consistente con su procedencia del fogón del piso de ocupación. Esta línea de análisis solamente permitió 


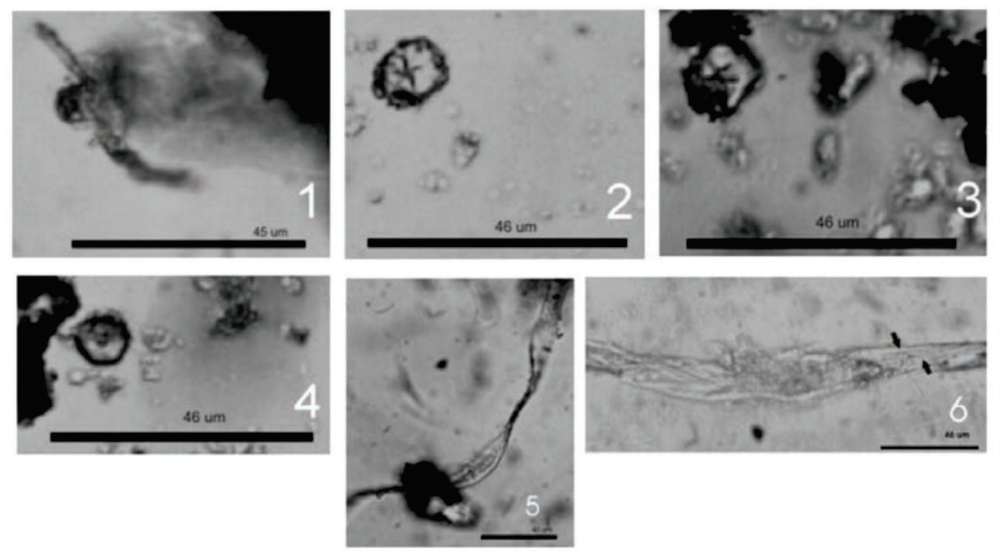

Figura 2. Granos de almidón de Zea mays, fibras de Gossypum sp. y tricomas de Nicotiana sp. observados bajo microscopio electrónico.

Figure 2. Zea mays starch grains, Gossypum sp. and trichomes of Nicotiana sp. observed under electron microscope.

obtener como dato que la muestra contenía una elevada presencia de elementos de la familia de las gramíneas, que abarca plantas potencialmente útiles como alimento -tanto de humanos como de otros animales-, como materia prima para la elaboración de mortero -uso arquitectónico-, como combustible, como parte del entorno, entre otras posibilidades.

La línea de evidencia que permitió identificar un listado más amplio de taxa vegetales fue el estudio de macrorrestos recuperados por la técnica de flotación, de sedimentos pertenecientes al piso de ocupación (Figura 3). Se identificaron taxonómicamente: Trichocereus sp., Prosopis spp. aff. flexuosa/nigra, Prosopis spp. aff. alba/chilensis, Zea mays, Opuntia sp., Geophroea decorticans, Chenopodium sp. Ch. quinoa var. quinoa, Ch. carnosulum, Solanaceae y Leguminosas (Petrucci 2017, Petrucci y Spano 2019).

Además, estos macrorrestos vegetales obtenidos por flotación, se analizaron según el grado de asociación que pudieron tener con las personas: silvestre, maleza, domesticado. Consideramos a las plantas silvestres como aquellas que crecen de manera natural y no son exitosas en espacios disturbados, las mismas pueden ser recolectadas o cultivadas (en sentido amplio o estricto). Las malezas, definidas a partir de su comportamiento ecológico, son plantas pioneras de sucesiones secundarias, exitosas en hábitats disturbados por los humanos y no requieren la intervención de éstos para su propagación. Estas últimas a su vez se diferencian en "ruderales", plantas que crecen en lugares donde habita el ser humano entre construcciones, ruinas o escombros, y "arvenses" que son plantas que crecen entre los cultivos (De Wet y Harlam 1975). Las prácticas de manejo en relación a las malezas pueden ser tolerancia, erradicación, cultivo o recolección (Lema 2009). Las plantas domesticadas requieren del cultivo y continua intervención del ser humano para su reproducción, ya que perdieron la habilidad de dispersar sus semillas o propágulos vegetativos de manera natural De Wet \& Harlan, 1975; Harlan, 1992). De esta manera, en el registro aparecieron sólo dos formas domesticadas, Zea mays (maíz) y Chenopodium quinoa var. quinoa (quinoa), y dos taxa silvestres, Prosopis spp. aff. flexuosal NOA de posibilidad de cultivo del algarrobo (Mc Rostie 2016), teniendo en cuenta los estudios en la región se lo considera como producto de la recolección (Petrucci 2017, Petrucci y Spano 2019).

En cuanto Chenopodium carnosulum si bien puede crecer por fuera de los campos de cultivo, también puede estar presente en los mismos, es por ello que puede ser considerada como una maleza facultativa (Petrucci 2017). Los ejemplares pertenecientes al género Chenopodium que no pudieron identificarse con mayor precisión, no se corresponden morfológica y anatómicamente con la Ch. quinoa var. quinoa ni con Ch. carnosulum. De esta manera, pueden ser formas malezoides o silvestres y por tanto pudieron estar sujetas a prácticas de recolección, tolerancia, erradicación, fomento o cultivo (sensu stricto).

En cuanto a las cactáceas por un lado tenemos los restos pertenecientes al género Opuntia que si bien hay varias que prosperan en el área todas sus formas son silvestre y se la considera como producto de la práctica de recolección. Por otro lado, existen trabajos donde consideran que los miembros del género Trichocereus podrían servir como indicadores de cambios climáticos pasados y uso de la tierra, a partir de su forma de crecimiento, longevidad y la tendencia a concentrarse en antiguas ruinas pre hispánicas (Halloy 2008) y en sitios perturbados por el establecimiento de poblaciones humanas actuales (Rodríguez-Arévalo et al. 2006). Es por esto que pueden considerarse como malezas facultativas no agrícolas (de tipo ruderal) que pudieron ingresar a la vivienda producto de prácticas de recolección, junto con las cosechas o con las heces utilizadas para la combustión.

A partir de análisis complementarios de procesamiento en macrorrestos de Zea mays, Chenopodium spp. y Prosopis spp. se hicieron aproximaciones a las posibles técnicas de procesamiento utilizadas para el consumo de estas especies. Los restos de maíz recuperados eran de tipo redondo, de endoperma vítreo, sin pericarpio y presentaban evidencia de cocción incompleta. Estas características posiblemente sean producto del hervido de los granos con cenizas para facilitar la eliminación del pericarpio y tengan que ver más con el pre-tratamiento 


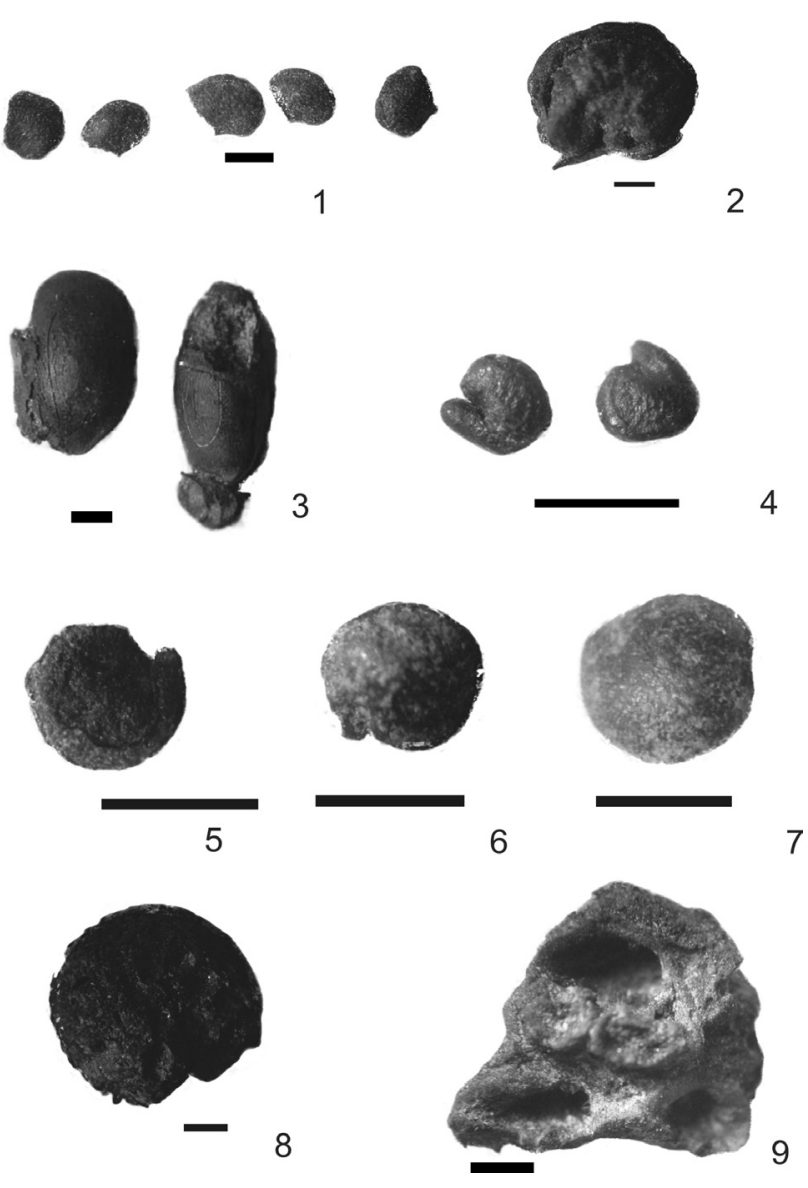

Figura 3. Carporrestos recuperados por la técnica de flotación del sitio Soria 2. 1. Trichocereus sp. 2. Opuntia sp. 3. Prosopis spp. 4. Solanaceae. 5. Quinoa. 6. Chenopodium carnosulum. 7. Chenopodium sp. 8. Zea mays (grano). Zea mays (marlo).

Figure 3. Remains recovered by the flotation technique of the Soria 2 site. 1. Trichocereus sp. 2. Opuntia sp. 3. Prosopis spp. 4. Solanaceae. 5. Quinoa. 6. Chenopodium carnosulum. 7. Chenopodium sp. 8. Zea mays (grain). 9. Zea mays (corn).

que con lograr una cocción completa para el consumo (Petrucci y Lema 2016). Ch. quinoa var. quinoa (quinoa), Ch. carnosulum y los especímenes que no pudieron identificarse a nivel de especie (grupo identificado como Chenopodium sp.), presentaron evidencias de desaponificación (Petrucci y López 2020). Las quinoas y las especies del género Chenopodium en general, producen un esteroide, la saponina, característica por su sabor amargo, su capacidad de generar espuma en una solución acuosa y por su toxicidad (hemolítico) (Romo et al. 2006; Vera et al. 2001). El proceso de desaponificación comprende una serie de técnicas básicas, y a medida que el mejoramiento avanza se pierden tejidos como el pericarpio. Esto constituye un rasgo de alto valor diagnóstico para aplicar en restos arqueobotánicos e inferir posibles prácticas post-cosecha y pre-cocción ya que la carbonización de los granos no provoca la desaparición de estructuras (López 2011).
Los endocarpos recuperados de Prosopis spp. pertenecen a las especies $P$. flexuosa o $P$. nigra (etnovariedad ${ }^{1}$ de algarrobo negro); los mismos presentan fisuras, posiblemente producto de la molienda. Las semillas correspondientes a las especies $P$. alba o $P$. chilensis (etnovariedad de algarrobo blanco) y poseían mesocarpo adherido, con aspecto brillante, lo cual podría indicar procesos de remojo y calor. De esta manera, se puede pensar que las señales de procesamiento nos hablan de molienda para la etnovariedad negra y posible elaboración de bebida -fermentada o sin fermentar- para la etnovariedad blanca (Petrucci y Capparelli 2020).

En la Tabla 1 se puede observar los restos identificados, la forma que se obtuvieron y las cantidades de ocurrencia de cada taxa.

Interrelaciones entre plantas y seres humanos: una perspectiva multiescalar

Toda práctica se encuentra histórica y espacialmente situada. Los seres humanos desarrollan su existencia de un modo localizado en múltiples contextos y relaciones, o, para decirlo en términos heideggerianos, son en el mundo (Dreyfuss 2002). Las prácticas situadas condensan una multiplicidad de conocimientos sobre el espacio circundante, percibido a través de la experiencia corporal, contextual e histórica.

Los materiales de origen vegetal cuya identificación compendiamos en este trabajo objetivan nociones y conceptos puestos en juego en pequeños actos cotidianos, cuya persistencia en el tiempo conforma los modos de hacer de una comunidad. Esas acciones conjugan la percepción que el sujeto posee de sí mismo, de sus redes sociales y del entorno (sin implicar necesariamente una escisión ontológica entre dichas percepciones). A partir de estas reflexiones surgió la propuesta de intentar una aproximación a las distintas escalas de interacción (entre sujetos y entre los sujetos y su entorno) a cuya luz puede leerse el estudio de la materialidad, en este caso, los restos de plantas utilizadas en asociación a la ocupación temprana del sitio Soria 2.

Para acercarnos a las interacciones sociales a distintas escalas tomamos como punto de partida el concepto de territorio propuesto por Giménez (2001) como el espacio del cual se apropia un determinado grupo social para asegurar su reproducción y necesidades vitales, ya sean materiales o simbólicas, que puede ser aprehendido en distintos niveles de escalas geográficas. Según el autor esta apropiación del espacio se da en dos dimensiones, una utilitaria o funcional (el espacio como medio de subsistencia y área geopolítica) y otra simbólico-cultural (lugar de desarrollo de la historia grupal, la tierra de los antepasados y referente de la identidad comunal).

\footnotetext{
1 Etnovariedad: clasificaciones que realizan los pobladores locales a partir de caracteres cuali y cuantitativos de las diferentes especies, las cuales muchas veces no tienen correspondencia con la clasificación Linneana.
} 


\begin{tabular}{|c|c|c|c|c|}
\hline Nombre & $\begin{array}{l}\text { Tipo de } \\
\text { vestigio }\end{array}$ & $\begin{array}{c}\text { Técnica de } \\
\text { identificación }\end{array}$ & $\begin{array}{l}\text { Modalidad de } \\
\text { recuperación }\end{array}$ & Frecuencia \\
\hline Trichocereus sp. & carporrestos & macroscópica & aislados & 132 \\
\hline $\begin{array}{l}\text { Geophroea } \\
\text { decorticans }\end{array}$ & carporrestos & macroscópica & aislados & 24 \\
\hline Chenopodium sp. & carporrestos & macroscópica & aislados & 15 \\
\hline Zea mays & $\begin{array}{l}\text { carporrestos } \\
\text { microrrestos }\end{array}$ & $\begin{array}{l}\text { macroscópica } \\
\text { microscópica }\end{array}$ & $\begin{array}{c}\text { aislados } \\
\text { hornillo de pipa }\end{array}$ & 14 \\
\hline $\begin{array}{c}\text { Chenopodium aff. } \\
\text { carnosulum }\end{array}$ & carporrestos & macroscópica & aislados & 13 \\
\hline Solanaceae & carporrestos & macroscópica & aislados & 9 \\
\hline $\begin{array}{c}\text { Chenopodium } \\
\text { quinoa var. quinoa }\end{array}$ & carporrestos & macroscópica & aislados & 8 \\
\hline Prosopis spp. & carporrestos & macroscópica & aislados & 6 \\
\hline aff. Gossypium sp. & microrrestos & microscópica & hornillo de pipa & 6 \\
\hline Opuntia sp. & carporrestos & macroscópica & aislados & 4 \\
\hline $\begin{array}{l}\text { Identificables tipo } \\
\text { leguminosas }\end{array}$ & carporrestos & macroscópica & aislados & 4 \\
\hline aff. Nicotiana sp. & microrrestos & microscópica & hornillo de pipa & 1 \\
\hline $\begin{array}{c}\text { Anadenanthera } \\
\text { colubrina var. cebil }\end{array}$ & $\begin{array}{c}\text { componentes } \\
\text { activos }\end{array}$ & química & hornillo de pipa & 1 \\
\hline Gramineas & microrrestos & microscópica & $\begin{array}{c}\text { Matriz } \\
\text { sedimentaria }\end{array}$ & 1 \\
\hline Total & & & & 237 \\
\hline
\end{tabular}

Tabla 1. Listado de ID de plantas, sitio Soria 2

Table 1. List of plant IDs, Soria 2 site

- y el territorio intermedio o región, correspondiente a aquel espacio intermediario entre aquel en el que se desarrollan las rutinas locales y el mundo. Sus límites son difusos; podemos pensarlos como aquellos ámbitos allende el espacio aldeano y su periferia, por fuera de los campos de cultivo y pastoreo, en donde se pueden desarrollar excursiones de cacería o búsqueda de materias primas y plantas que no crecen localmente, por ejemplo.

En tanto espacio apropiado, entonces, el territorio tendría una condición multiescalar: puede ser percibido por los sujetos en niveles distintos y superpuestos de la escala geográfica, desde la vivienda hasta el mundo. En su definición posee un peso importante la percepción por parte del sujeto. Este énfasis teje lazos con la definición de Tilley (2004) de paisaje, es decir, un conjunto de relaciones percibidas entre lugares, los sentires de las personas, el habitar, los movimientos y las prácticas en un espacio geográfico, que puede o no exhibir límites topográficos reconocibles. Justamente esta condición subjetiva de la definición de territorio y de paisaje ofrece flexibilidad al momento de evaluar los alcances de cada nivel propuesto por Giménez 2001 para nuestro caso de estudio.

Ensayando y adaptando dicho modelo, podemos pensar a Soria 2 en múltiples escalas:

- $\quad$ el territorio más íntimo e inmediato de la vivienda o casa habitación: el ámbito en el que se desarrollan actividades propias de la vida cotidiana entre coresidentes. Quizás se trate de la escala en la cual los límites son más reconocibles físicamente, dados por los muros de la casa que separan interior y exterior;

- $\quad$ el territorio próximo del asentamiento aldeano o nivel local donde se llevan a cabo las interacciones sociales cotidianamente entre co-residentes de distintas viviendas, y con el cual se construye un vínculo afectivo de pertenencia en tanto prolongación de la casa. Puede corresponder a las vías de circulación al interior del asentamiento, a los espacios de uso comunitario -como ser terrenos destinados a actividades de producción de alimentos-, en definitiva, a los lugares percibidos como objetivación del colectivo social;
Dado que el planteo de Giménez procede de la Geografía Social y por ende lo aplica a sociedades actuales, incluye también un nivel más abarcativo, el de los Estado-nación, en el cual si bien predominan criterios políticos y jurídicos en la definición del territorio, subyace la pertenencia simbólica del sujeto a su comunidad nacional. No es posible establecer una correspondencia a partir de esta definición para un caso arqueológico de tiempos pre-incaicos, por lo cual tomaremos este nivel en su dimensión espacial en tanto escala extra-regional, para aludir al mundo por fuera de los límites percibidos físicos y/o simbólicos de la región.

A estas escalas, las cuales, reiteramos, no son meramente materiales sino que se entrelazan con la percepción humana acerca del entorno y de la interrelación con los demás, podemos superponer un nivel correspondiente al ámbito metafísico de las creencias, las maneras de entender el mundo y en particular a las plantas, que intersecta los distintos niveles escalares.

En la Tabla 2 se sintetizan las prácticas que involucraron a los restos vegetales recuperados por distintas técnicas, incluyendo otras materialidades que pudieron estar asociadas, y las escalas espaciales posibles para la interacción entre plantas y humanos. También se informa la índole de la procedencia de los materiales analizados.

Cada uno de los hallazgos nos habla de prácticas cotidianas al interior de la casa (Figura 4. 1), comenzando por la alimentación. La ingesta de alimentos -líquidos o sólidos- es un acto que trasciende la necesidad vital de los seres humanos en tanto organismos, y juega un papel clave en la socialización de las personas en la sucesión acumulativa de eventos cotidianos en los que además 


\begin{tabular}{|c|c|c|c|}
\hline $\begin{array}{l}\text { Planta identificada } \\
\text { Nombre científico }\end{array}$ & $\begin{array}{c}\text { Materialidades directa o } \\
\text { indirectamente halladas } \\
\text { en el sitio }\end{array}$ & Prácticas inferidas & $\begin{array}{c}\text { Escalas posibles de } \\
\text { interacción } \\
\text { planta-humano }\end{array}$ \\
\hline Zea mays & $\begin{array}{l}\text { Morteros, molinos, manos, } \\
\text { recipientes cerámicos }\end{array}$ & $\begin{array}{l}\text { Procesamiento. } \\
\text { Cocción.Ingesta. } \\
\text { Cultivo }\end{array}$ & $\begin{array}{l}\text { La vivienda } \\
\text { El territorio próximo } \\
\text { o local }\end{array}$ \\
\hline Prosopis spp. & $\begin{array}{l}\text { Morteros, molinos, manos, } \\
\text { recipientes cerámicos }\end{array}$ & $\begin{array}{l}\text { Recolección. } \\
\text { Procesamiento. } \\
\text { Cocción. Ingesta }\end{array}$ & $\begin{array}{l}\text { La vivienda } \\
\text { El territorio próximo } \\
\text { o local }\end{array}$ \\
\hline Gossypium sp. & Pipas & $\begin{array}{l}\text { Hilado. Teñido. } \\
\text { Cultivo } \\
\text { ¿Limpieza de } \\
\text { hornillos de pipa? } \\
\text { ¿Utilización de } \\
\text { contenedores? }\end{array}$ & $\begin{array}{l}\text { La vivienda } \\
\text { El territorio próximo } \\
\text { o local }\end{array}$ \\
\hline $\begin{array}{l}\text { Anadenanthera } \\
\text { colubrina var. cebil }\end{array}$ & Pipas & $\begin{array}{l}\text { Ingesta. Vinculación } \\
\text { con la esfera extra } \\
\text { sensorial }\end{array}$ & $\begin{array}{l}\text { La vivienda } \\
\text { El territorio próximo } \\
\text { o local } \\
\text { El territorio extra } \\
\text { regional } \\
\text { Interacción con la } \\
\text { esfera trascendental }\end{array}$ \\
\hline $\begin{array}{l}\text { Chenopodium } \\
\text { quinoa var. quinoa }\end{array}$ & Recipientes cerámicos & $\begin{array}{l}\text { Procesamiento. } \\
\text { Cocción. Ingesta }\end{array}$ & $\begin{array}{l}\text { La vivienda } \\
\text { El territorio próximo } \\
\text { o local }\end{array}$ \\
\hline Trichocereus sp. & & $\begin{array}{l}\text { Recolección. } \\
\text { Ingesta }\end{array}$ & \begin{tabular}{|l|} 
La vivienda \\
El territorio próximo \\
o local \\
\end{tabular} \\
\hline $\begin{array}{l}\text { Geophroea } \\
\text { decorticans }\end{array}$ & $\begin{array}{l}\text { Morteros, molinos, } \\
\text { manos, recipientes } \\
\text { cerámicos }\end{array}$ & $\begin{array}{l}\text { Recolección. } \\
\text { Procesamiento. } \\
\text { Cocción.Ingesta }\end{array}$ & $\begin{array}{l}\text { La vivienda } \\
\text { El territorio próximo } \\
\text { o local }\end{array}$ \\
\hline Chenopodium sp. & Recipientes cerámicos & $\begin{array}{l}\text { Recolección, } \\
\text { tolerancia, } \\
\text { erradicación, } \\
\text { fomento o cultivo. } \\
\text { Procesamiento. } \\
\text { Cocción. Ingesta } \\
\end{array}$ & $\begin{array}{l}\text { La vivienda } \\
\text { El territorio próximo } \\
\text { o local }\end{array}$ \\
\hline Ch. carnosulum & ¿Recipientes cerámicos? & Tolerancia. Ingesta & $\begin{array}{l}\text { La vivienda } \\
\text { El territorio próximo } \\
\text { o local }\end{array}$ \\
\hline Opuntia sp. & & Recolección. Ingesta & $\begin{array}{l}\text { La vivienda } \\
\text { El territorio próximo } \\
\text { o local } \\
\end{array}$ \\
\hline Solanaceae & & Recolección. Ingesta & $\begin{array}{l}\text { La vivienda } \\
\text { El territorio próximo } \\
\text { o local }\end{array}$ \\
\hline
\end{tabular}

de preparar y compartir la comida, se fijan experiencias y hábitos, se transmite y recibe información, y se incorporan modos de hacer que se transforman en rutinas (Atalay y Hastorf 2006). Y también resultan evidencia de las etapas involucradas en la secuencia de producción de alimentos que tuvieron lugar en el ámbito de la casa y sus acciones asociadas (Goody 1995):

procesamiento: las evidencias directas de la presencia de almidones de maíz y frutos de algarrobo negro, sumado al registro de implementos de molienda -molinos de mano, morteros, conanas y manos de moler elaborados en piedra-, dan cuenta de un contexto de práctica de molienda individual-familiar (Carbonelli 2009). Las señales de desaponifiación advertidas en los restos de quinoa constituyen también un marcador de la ocurrencia de acciones de lavado previas a la cocción de manera de posibilitar la ingesta. En esta etapa incluimos también el probable hervido de maíz para el pelado de los granos y el posible remojo y calor aplicado al algarrobo blanco.

cocción: las especies mencionadas en el ítem anterior requieren ser sometidas a calor para ser transformadas en alimentos. En el sitio se
Tabla 2. Vinculación entre plantas identificadas (con sus nombres vulgares en aquellos casos en que pudo identificarse taxón), otras materialidades, posibles prácticas asociadas y escala de acción propuesta, sensu Giménez 2001.

Table 2. Linkage between identified plants (with their vulgar names in those cases in which taxon could be identified), other materialities, possible associated practices and proposed scale of action, sensu Giménez 2001.

han encontrado asociadas al piso de ocupación fragmentos de ollas con evidencias de haber estado sometidas a calor (Baigorria Di Scala 2009), y que pudieron ser contenedores utilizados para hervido o guisado. También se hallaron fragmentos de cucharas, elaboradas en cerámica, aptas para la manipulación de los preparados (Spano 2011). El fogón, ubicado en la esquina sureste del patio de la casa, ofreció las condiciones de infraestructura tecnológica para la cocción de los ingredientes.

ingesta: el registro cerámico asociado al piso de la casa incluye fragmentos de platos, cuencos y escudillas, plausibles por su morfología de haber sido utilizados para servicio de los alimentos sólidos y líquidos (Baigorria Di Scala 2009; Spano 2011).

Por otra parte, los restos de fibras de algodón hallados en las adherencias carbonizadas al interior de pipas, uno de ellos con marcas tecnológicas y coloreado, dan cuenta de la utilización de hilados. Los contenidos identificados en las pipas cerámicas -cebil y, posiblemente, una variedad silvestre de tabaco- refieren a las prácticas fumitorias que se desarrollaron en el ámbito de la casa, y a la realización de rituales íntimos en contextos domésticos familiares, acotados espacialmente (Palamarczuk et al. 2007).

Este listado de prácticas inferidas ofrece una pintura de situaciones que construyeron la cotidianidad de las personas en el entorno más inmediato de la interacción de los agentes, ya sea entre humanos, como entre éstos y las plantas.

La definición de los límites de lo que en términos de Giménez (2001) sería el territorio próximo no es sencilla, en el caso de la MAB. Nominalmente se corresponde con el espacio aldeano, es decir, el área que incluye a la casa y que fue percibida como el ámbito para la interacción con los residentes de otras viviendas que conformaban el colectivo social de pertenencia. Ahora bien, no es sencillo reconocer en superficie asentamientos 

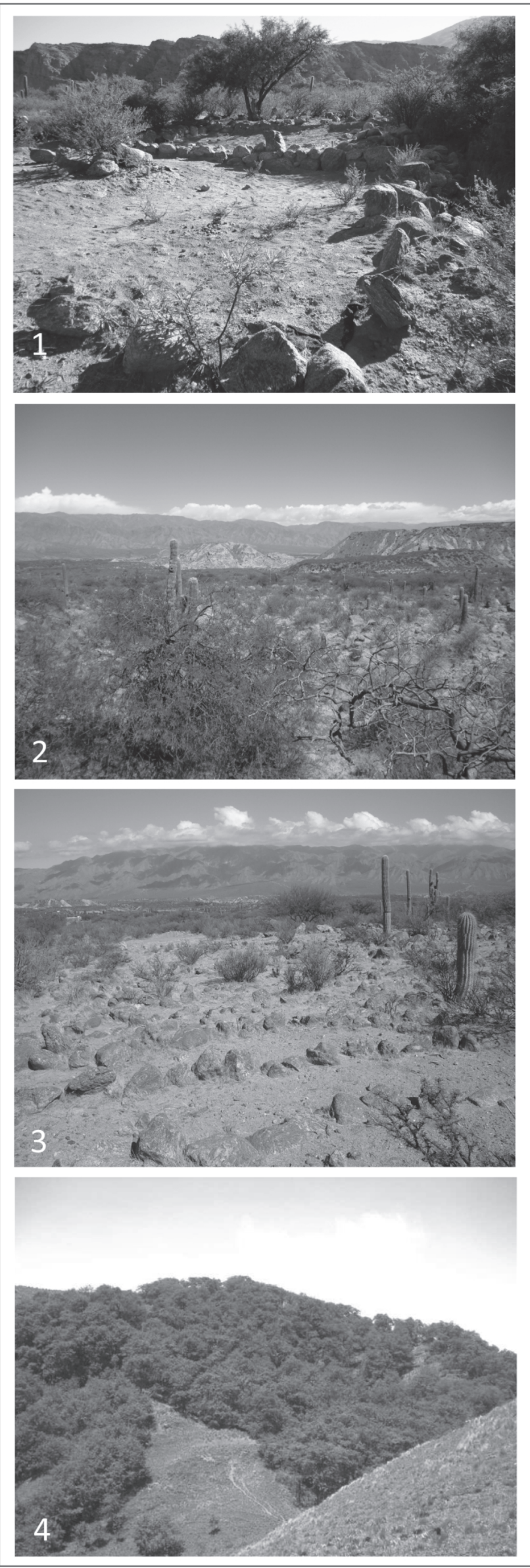

Figura 4. Territorios inferidos. 1. Recintos de Soria 2, escala de la vivienda. 2. Vista general del emplazamiento de Soria 2 en el contexto de la MAB, escala de los territorios próximos (fotografía: Alina Álvarez Larrain). 3. Detalle de campos con estructuras agrícolas en la MAB, escala de los territorios próximos (modificada de Álvarez Larrain 2016, Fig. 7). 4. Ecorregión de las Yungas, escala del territorio extra regional (tomada de Equipo IDECAT S/D).

Figure 4. Inferred territories. 1. Structures of Soria 2, housing scale. 2. Panoramic view of the Soria 2 site in the context of the $M A B$, neighboring territories scale (photography: Alina Álvarez Larrain). 3. Detail of fields with agricultural structures in the MAB (modified image from Álvarez Larrain 2016, Fig. 7). 4. Yungas ecological region (taken image from Equipo IDECAT S/D).

tempranos en el valle de Yocavil, por razones ampliamente expuestas en la literatura (e. g. Scattolin 2000). Entre otras razones, la persistente ocupación humana durante los últimos mil años ha ocasionado un «palimpsesto acumulativo»y «multi-temporal» (Bailey 2007; Olivier 1999), en el cual se superponen los restos materiales de distintas dinámicas sociales del pasado, en cuanto a arquitectura y material cerámico en superficie. Teniendo en consideración la distribución de cerámica de estilos tempranos y la presencia de unidades arquitectónicas con muros de morfología y tipo de planta semejantes a Soria 2, se ha propuesto una superficie mínima de 35 hectáreas de ocupación temprana en el este del glacis o terrazas de piedemonte (Álvarez Larrain 2015). La excavación del sitio Soria 3, emplazado 300 metros al noroeste de Soria 2, permitió bosquejar una modalidad local de hábitat temprano en base a semejanzas en el trazado de planta, tecnología constructiva, materialidad cerámica y lítica, y en cuanto a prácticas de entierro de infantes post abandono (Álvarez Larrain et al. 2017). En cuanto a los espacios destinados al cultivo, hasta el momento no se han podido definir áreas productivas exclusivas para tiempos tempranos. La MAB se erige en un ambiente de mesadas surcado por los principales cauces tributarios del río Santa María que nacen en la cumbre de la Sierra del Aconquija, y propician una suerte de entorno de "oasis fértiles", en contraste con el ambiente más árido del valle principal (Tineo 2005). Las prospecciones emprendidas en Andalhuala han permitido registrar arquitectura y estilos alfareros semejantes a Soria 2 y 3 en la margen sur del río Azampay (Álvarez Larrain 2018). Estos espacios parecen haber formado parte del asentamiento humano durante el primer milenio $A D, y$ es esperable que se hayan destinado terrenos para fines productivos en estos glacis bajos, dadas las condiciones en términos de calidad de suelos, pendiente -moderada-, posibilidad de insolación para los cultivos y accesibilidad al agua para riego, con una inversión tecnológica requerida moderada (Álvarez Larrain 2018). En vistas de esto, aunque por el momento no se ha podido dar con datos directos, los campos de cultivo pudieron estar dispersos 
en íntima vinculación con las unidades residenciales, o bien ubicados en los terrenos bajos del fondo de valle, donde hoy se concentra el pueblo actual de Andalhuala (Álvarez Larrain 2018). Debe tenerse en cuenta, para fortalecer estas especulaciones, que en tiempos tardíos una gran parte de las unidades arquitectónicas se destinaron a fines agrícolas (Álvarez Larrain 2015, 2016): terrazas y recintos de siembra debieron ser empleados para cultivos mesotérmicos, como maíz, zapallo y ají; se detectaron también acumulaciones longitudinales de piedras producto de tareas de despeje de la superficie para la siembra y tramos empedrados de canales y acequias (Álvarez Larrain 2018). Esta sucesiva intervención deviene en un paisaje sedimentado (Figura 4.2).

A partir de esta descripción interpretativa de las condiciones ecológicas y de la evidencia con la que se cuenta para tiempos tardíos, podemos pensar en que el territorio próximo, en términos de Giménez (2001), fue el espacio destinado a las tareas agrícolas, en el que la interacción entre personas y plantas se objetivó en la obtención de productos bioculturales como el maíz y la quinoa. Pero también fue el espacio conocido y recorrido diariamente para el encuentro con otras personas, interactuar con los animales, buscar rocas para la talla y arcilla para hacer vasijas, y también para recolectar frutos y hojas de plantas silvestres (Prosopis spp., Geoffroea decorticans, Chenopodium sp., entre otras); donde se fomentó el crecimiento de ciertas plantas, se las dejó crecer o se las eliminó. Es el territorio de los algarrobos y las cactáceas. Los límites físicos de este espacio no son asequibles a nuestra percepción, pero sin duda formó parte del mismo la superficie de la MAB (Figura 4.3).

El territorio intermedio o región, aquel espacio que se percibe más allá del ámbito de asentamiento del colectivo social y sus alrededores, está representado en el hallazgo de las fibras de algodón. Las especies pertenecientes al género Gossypium no soportan las heladas, razón por la cual su distribución se limita a latitudes tropicales y subtropicales, y su ciclo de crecimiento anual está vinculado a las estaciones secas y húmedas; es por ello que la mayoría de los algodones americanos crecen en los trópicos más áridos, donde un verano húmedo alterna con invierno seco (Stephens 1975). Si bien el análisis de fibras no alcanzó la determinación a nivel específico, probablemente se trate de restos de G. barbadense, la única especie de distribución en Sudamérica cuyo centro de variabilidad se encuentra en los valles andinos, y su centro de origen en los valles fértiles de la costa del Perú (Carnevali 1970). El clima de los valles de la actual provincia de Catamarca fue particularmente adecuado para el cultivo del algodón, llegando a ser una de las principales producciones de ese territorio en la época colonial y republicana temprana (Sánchez de Oviedo 1937). Hasta el momento no se han conocido otros registros de hallazgos de algodón en el valle de Yocavil, para tiempos prehispánicos; si bien no se puede descartar su cultivo en la región, dadas las características de los ambientes óptimos para que prospere la especie, es probable que su procedencia se relacione con áreas de mayor humedad, como por el Valle de Catamarca por ejemplo, distante a unos $200 \mathrm{~km}$ hacia el sur. Eso implicaría la interacción con poblaciones más alejadas, ya sea en la forma de intercambios como de otras estrategias desconocidas. De todas formas, no puede descartarse del todo su procedencia de territorios próximos.

Ya por fuera del territorio a escala de la región o a escala extra regional (Figura 4.4), el hallazgo de restos de cebil en una de las pipas del sitio nos remite a los pisos inferiores de la provincia fitogeográfica de las yungas como ámbito de procedencia. Por ejemplo, los cebilares de la Sierra del Ambato se encuentran a unos $130 \mathrm{~km}$ en dirección sur. Este acceso a un recurso tan apreciado, en virtud de su uso ritual como alucinógeno, implicó, para las personas que habitaron la MAB, el acceso al conocimiento acerca de la zona de crecimiento de estos árboles, la vinculación con poblaciones distantes y el desplazamiento de personas para el traslado del producto. Esta interacción necesariamente debió basarse en el conocimiento interpersonal, el intercambio de experiencias y en la reproducción de la memoria colectiva.

El caso del consumo de la planta de cebil en pipas es un ejemplo de cómo la perspectiva multiescalar atraviesa objetos y situaciones mediantes las prácticas. Se produjo el acto ritual de fumar en el ámbito doméstico (escala de la vivienda); la elaboración y utilización de pipas, así como las recetas de las mezclas fumitorias, constituyen un conocimiento que excede lo local, teniendo en cuenta el uso extendido de pipas de fumar en el NOA para tiempos tempranos, (escala de interacción de los territorios próximos); para la limpieza de las pipas se pudo utilizar hisopos con punta de algodón, o tal vez se guardó la pipa y/o la mezcla fumitoria en un contenedor confeccionado con fibras de algodón (escala de los territorios intermedios). El cebil consumido es una especie que crece por fuera de la región del valle (escala extra regional), y a su vez, la utilización de esta planta en la performance de fumar en pipa debió generar dos elementos considerados como partes importantes del ritual: la producción de humo y efectos alucinatorios, merced a los componentes psicoactivos del cebil, que permitían entrar en contacto con la esfera de lo sobrenatural (Planella et al. 2012). De este modo, podemos plantear que en la asociación entre la utilización de la pipa 1 y las plantas que componen el fumitorio vemos un ejemplo de cómo se manifiesta la perspectiva multiescalar en tanto percepción, alcanzando incluso el ámbito inmaterial.

\section{Algunas reflexiones hacia una narrativa arqueológica del uso de plantas en Andalhuala Banda}

En este artículo intentamos recuperar información 
producida a lo largo de varios años relativa a la identificación de vegetales en Soria 2, reunirla y ponerla en juego con otros datos contextuales para proponer narrativas arqueológicas acerca del uso de plantas en el pasado. Las relaciones entre personas y plantas fueron abordadas a partir de la identificación taxonómica mediante distintas vías de análisis, tomando en consideración las alteraciones antrópicas que los restos pudieran exhibir para identificar gestos técnicos (carbonizado, remoción de tejidos mediante limpieza, marcas tecnológicas, etc.) y en paralelo, teniendo en cuenta la información contextual construida a partir de otros registros materiales y del estado de conocimiento acerca del tema. La perspectiva arqueo-etnobotánica, para delinear las relaciones entre los seres humanos y las plantas en el pasado, y multiescalar, para aprehender la materialidad a distintas escalas a la vez, nos permitió esbozar un conjunto de prácticas que intersectan personas y plantas.

Esta dimensión multiescalar imbricada en los restos arqueológicos, creemos, puede complementarse con el planteo de autoras como Browne Ribeiro (2010) o Tringham (1994), quienes han propuesto la idea de una narración arqueológica centrada en los sujetos y abordar el estudio de los lugares desde la perspectiva de las personas, sin abandonar los intentos de acercarnos a la intencionalidad y los significados,

¿Cómo relatar una historia local de las plantas y las personas en Soria 2? Los restos analizados provienen de un contexto doméstico. Las personas que los generaron habitaron la vivienda, posiblemente vinculadas a través de relaciones de parentesco. Al espacio al que hoy llamamos Soria 2 los sujetos ingresaron productos bioculturales como maíz y quínoa, obtenidos en tanto resultantes del trabajo entre pares en el territorio aldeano para acompañar e intervenir en el ciclo de crecimiento de las plantas. Los partieron, molieron, limpiaron y los tornaron comestibles para su ingesta y la de otros. También trajeron frutos extraídos de plantas de las cercanías del asentamiento y más allá, que fueron transformados en alimentos. Aplicaron en estas acciones años de conocimientos transmitidos y experiencias compartidas. Es en estas acciones cotidianas, atravesadas por intencionalidades, decisiones y sentidos, que se fue construyendo la reproducción social.

Las plantas fueron observadas, reconocidas, sistematizado el conocimiento relativo a ellas y transmitido entre generaciones. Fueron instadas a reproducirse, cosechadas, recolectadas o segadas. Fueron buscadas, portadas, trasladadas y recibidas. Fueron cuidadas y consideradas parientes -basta pensar en el concepto de familia extensa andina (Álvarez Larrain et al. 2016). Fueron seccionadas, lavadas, molidas, hervidas, tostadas e ingeridas. Participaron de la rutina del alimento diario y de la eventualidad del regocijo y el encuentro.
Provocaron estados alterados de la consciencia y fueron un canalizador para aventurarse entre mundos.

Claramente, estas páginas contienen un relato incompleto y parcial. Incompleto, por las características propias del registro arqueológico; y parcial, por el uso instrumental de categorías biológicas o agronómicas como "malezas", "tolerancia" o "doméstico", que inevitablemente imponen límites y connotaciones para una aproximación totalizadora, como también se encuentran limitadas las categorías arqueológicas y geográficas -"territorios próximos", "aldea", "región"-. Como señala Descola (2010), existe diversidad de culturas, y también diversidad de naturalezas. Los cuerpos condensan cientos de miles de años de sucesivas historias locales entre personas y plantas. La que aquí esbozamos es una más de ellas.

Buenos Aires, 15 de mayo de 2020.

\section{Agradecimientos}

A Myriam Tarragó, por su apoyo y orientación en las distintas instancias de nuestras investigaciones. A Valeria Palamarczuk, por su persistencia para dar forma al "proyecto flotación". A Verónica Lema y María Lelia Pochettino por su formación constante en el estudio de los restos vegetales. A Alina Álvarez Larrain por brindarnos su apoyo con las imágenes. Y a los revisores anónimos por sus contribuciones.

\section{Bibliografía}

Álvarez Larrain, A. (2015). Habitar una región. Espacialidad arquitectónica y construcción de paisajes en Andalhuala, valle de Yocavil (Catamarca, Argentina). Tesis doctoral en Arqueología, Facultad de Filosofía y Letras, Universidad de Buenos Aires.

Álvarez Larrain, A. (2016). Paisajes agroalfareros del primer y segundo milenio DC en la Terraza de Andalhuala Banda (Yocavil, noroeste argentino). Ñawpa Pacha. Journal of Andean Archaeology, 36 (2), 161-184.

Álvarez Larrain, A. (2018). El devenir histórico de los paisajes culturales de un pueblo catamarqueño. (Andalhuala, Yocavil, Argentina). Revista Colombiana de Antropología, 54(1), 219-252.

Álvarez Larrain, A., Spano, R. C. y Grimoldi, M. S. (2016). Soria 3: nuevas evidencias de la ocupación aldeana temprana en Yocavil, Noroeste Argentino. Un ejercicio interpretativo. Revista Española de Antropología Americana, 46, 219-239.

Álvarez Larrain, A., Spano, R. C. y Grimoldi, M. S. (2107). Ollas como urnas, casas como tumbas: reflexiones en torno a las prácticas de entierro de infantes y niños pequeños en tiempos tempranos (Andalhuala Banda, 
sur de Yocavil). Comechingonia. Revista de Arqueología, 21(1), 39-70.

Andreoni, D., Spano, R. C. y Lema, V. (2012). Nota sobre evidencias de uso de plantas en el sitio Soria 2 a partir del análisis microscópico del contenido de pipas. Arqueología, 18, 235-243.

Atalay, S. y Hastorf, C. (2006). Food, Meals, and Daily Activities: Food Habitus at Neolithic Çatalhöyük. American Antiquity, 71(2), 283-319.

Baigorria Di Scala, L. J. (2009). El sitio Formativo Soria 2: Estudio Tecno Morfológico del conjunto cerámico ordinario. [Tesis de grado inédita. Facultad de Filosofía y Letras, Universidad de Buenos Aires. 117 páginas. Museo Etnográfico Juan B. Ambrosetti].

Bailey, G. (2007). Time Perspectives, Palimpsests and the Archaeology of Time. Journal of Anthropological Archaeology, 26(2), 198-223.

Browne Ribeiro, A. (2010). Viviendo en el pasado: elaboración de una biografía multisensorial de la gente y los lugares en Amazonia Central. En Hermo,D. y Miotti, L. (Eds.), Biografías de Paisajes y Seres: Visiones desde la Arqueología Sudamericana (pp. 37-45). Córdoba, Argentina: Encuentro Grupo Editor.

Cabrera, A. L. (1971). Fitogeografía de la República Argentina. Boletín de la Sociedad Argentina de Botánica, 14(1-2).

Carbonelli, J. P. (2009). Motivos porque y para en la tecnología de un sitio formativo en el Valle de Yocavil (Pcia. de Catamarca). Intersecciones en Antropología, $12,31-44$.

Carnevali, R. (1970). Variación geográfica y origen de Gossypium barbadense en Argentina. Boletín de la Sociedad Argentina de Botánica, 11(suplemento), 277285.

Descola, P. (2010). Diversité des Natures, Diversité des Cultures. Les Petites Conférences. París, Francia: Bayard.

De Wet, J. M. y Harlan, J. R. (1975). Weeds and Domesticates: evolution in the man-made hábitat. Economic Botany, 29, 99-107.

Dreyfuss, H. 2002. Ser-en-el-Mundo. Comentario a la División I de Ser y Tiempo de Martín Heidegger. Santiago de Chile: Chile: Cuatro Vientos.

Giménez, G. (2001). Cultura, territorio y migraciones. Aproximaciones teóricas. Alteridades, 11(22), 5-14.

Goody, J. (1995). Cocina, cuisine y clase. Estudio de sociología comparada. Barcelona, España: Gedisa.

Halloy, S. (2008). Crecimiento exponencial y supervivencia del cardón (Echinopsis atacamensis subsp. pasacana) en su límite altitudinal (Tucumán, Argentina). Ecología en Bolivia, 43(1), 6-15.

Harlan, J. (1992). Crops and man. Nueva York, Estados Unidos: American Society of Agronomy.

Lema, V. (2009). Domesticación Vegetal y Grados de Dependencia Ser Humano-Planta en el Desarrollo Cultural Prehispánico del Noroeste Argentino. Tesis doctoral inédita, Facultad de Ciencias Naturales y Museo, Universidad Nacional de La Plata.

López, María L. (2011). Estudio de Macro y Micro Restos de Quínoa de Contextos Arqueológicos del Último Milenio en Dos Regiones Circumpuneñas. Tesis doctoral inédita, Facultad de Filosofía y Humanidades, Universidad Nacional de Córdoba.

Maffi, L. (2001). Introduction. On the Interdependence of Biological and Cultural Diversity. En L. Maffi (Ed.), On Biocultural Diversity.Linking Language Knowledge and the Environment (pp.1-50). Washington, Estados Unidos: Smithsonian Institute Press.

McRostie, V. (2016). Algarrobales de Atacama. ¿Nativos o exóticos? ¿silvestres o domésticos? Actas del XIX Congreso Nacional de Arqueología Argentina, Serie Monográfica y Didáctica (54), 1539-1544.

Nazarea, V. (Ed.). (1999). Ethnoecology. Situated knowledge/located lives. Tucson, Estados Unidos: University of Arizona Press.

Olivier, L. (1999). The Hochdorf 'Princely' Grave and the Question of the Nature of Archaeological Funerary Assemblages. En T. Murray (Ed.), Time and Archaeology (pp. 109-138). Londres, Inglaterra: Routledge.

Palamarczuk, V., Spano, R., Weber, F., Magnifico, D., López, S. y Manasiewicz, M. (2007). Soria 2. Apuntes sobre un Sitio Formativo en el Valle de Yocavil (Catamarca, Argentina). Intersecciones en Antropología, 8, 121-134.

Planella, M. T., Belmar C., P., Quiroz L., L. y Estévez G., D. (2012). Propuesta integradora para el estudio del uso de plantas con propiedades psicoactivas en pipas del Período Alfarero Temprano y sus implicancias sociales. Revista de Antropología 25(1 semestre), 93-119.

Petrucci, N. (2017). Complejidad social y diversidad biocultural en el valle de Yocavil: mil quinientos años de interacciones entre comunidades humanas y poblaciones vegetales. Tesis doctoral inédita, Facultad de Ciencias Naturales y Museo, Universidad Nacional de La Plata. 
Petrucci, N. y Capparelli, A. (2020). Análisis de las técnicas de procesamiento en restos de Prosopis sp. recuperados de los sitios Soria 2 y Rincón Chico 15 de la provincia de Catamarca. Ms.

Petrucci, N. y Lema, V. (2016). Caracterización de procesamiento y carbonización de productos derivados de Zea mays L.: aproximación experimental aplicada a restos arqueobotánicos carbonizados. Intersecciones en Antropología, 17, 291-302.

Petrucci, N. y López, M. L. (2020). Interpretación de posibles modalidades de procesamientos en restos carbonizados del género Chenopodium recuperados del sitio Soria 2 (Catamarca, Argentina). Latin American Antiquity. Ms.

Petrucci, N. y Spano, R. C. (2019). Arqueobotánica del sitio temprano Soria 2 (Catamarca, Argentina). Boletín de la Sociedad Argentina de Botánica, 54(1), 137-154.

Rodríguez-Arévalo, I., Casas, A., Lira, R. y Campos, J. (2006). Uso, manejo y procesos de domesticación de Pachycereus hollianus (F.A.C. Weber) Buxb. (Cactaceae), en el valle de Tehuacán-Cuicatlán, México. Revista Interciencia, 31(9), 677-685.

Romo, S., Rosero, A., Forero, C. y Cerón, E. (2006). Nutritional potencial of quinua flour (Chenopodium quinoa Willd) piartal variety in Colombian Andes. Parte Uno. Facultad de Ciencias Agropecuarias, 4(1), 112-125.

Rosso, C. y Spano, R. C. (2005-2006). Evidencias del uso de alucinógenos en pipas halladas en dos sitios tempranos de los valles Calchaquíes. Arqueología, 13, 79-98.

Sánchez Oviedo, C. (1937). El algodón. Factor importante en la historia de Catamarca. Junta Nacional del Algodón. Publicación 24, 5-45. Buenos Aires, Argentina: Ministerio de Agricultura.
Scattolin, M. C. (2000). Santa María durante el Primer Milenio A.D. ¿Tierra baldía? Etnografiska Museet $i$ Goteborg. Arstryck, 1995-1998, 63-83.

Stephens, S. (1975). Some observations on Photoperiodism and the development of annual forms of domesticated cottons. Economic Botany, 30, 409-418.

Spano, R. C. (2011). Primera sistematización de las características estilísticas de la alfarería fina del sitio Soria 2 (Valle de Yocavil, Noroeste argentino). Revista del Museo de Antropología, 11, 127-144.

Spano, R. C., Grimoldi, M. S. y Palamarczuk, V. (2014). Morir temprano. Entierros de infantes en un espacio doméstico formativo de Santa María, noroeste argentino. Estudios. Antropología. Historia. Nueva Serie, 2, 141173.

Tilley, C. (2004). The Materiality of Stone: Explorations in Landscape Phenomenology. Oxford, Inglaterra:.Berg.

Tringham, R. (1994). Engendered places in Prehistory. Gender, Place and Culture, 1(2), 169-203.

Tineo, A. 2005. Estudios Hidrogeológicos del Valle del Río Santa María - Provincia de Catamarca - Serie Correlación Geológica 20. San Miguel de Tucumán, Argentina: Instituto Superior de Correlación Geológica (INSUGEO).

Vera A., Vargas, M. y Delgado, G. (2001). Actividad biológica de las saponinas de la quínoa (Chenopodium quinoa Willd.). Journal Boliviano de Ciencias, 3(6). www. univalle.edu/publicaciones/journal

Zucol, A. (2005). Prospección y análisis de micro-restos en muestras sedimentarias y fragmentos de cerámicas de sitios arqueológicos de la provincia de Catamarca (Argentina). Ms. 